\title{
Early warning flood detector adopting camera by Sobel Canny edge detection algorithm method
}

\author{
Satryo B. Utomo ${ }^{1}$, Januar Fery Irawan ${ }^{2}$, Rizqi Renafasih Alinra ${ }^{3}$ \\ ${ }^{1,3}$ Department of Electrical Engineering, Universitas Jember, Indonesia \\ ${ }^{2}$ Department of Mining Engineering, Universitas Jember, Indonesia
}

\section{Article Info \\ Article history: \\ Received Mar 5, 2020 \\ Revised Mar 24, 2021 \\ Accepted Mar 30, 2021 \\ Keywords: \\ Canny edge detection \\ Computer vision \\ Flood detection \\ Image processing \\ Sobel edge detection \\ Water level}

\begin{abstract}
Early warning of floods is an essential part of disaster management. Various automatic detectors have been developed in flood mitigation, including cameras. But reliability and accuracy have not been improved. Besides, the use of monitoring devices has been employed to monitor water levels in various water building facilities. The early warning flood detector was carried out with a sensor camera using an orange ball that floats near the water level gauge in a bounding box. This approach uses the integration of computer vision and image processing, namely digital image processing techniques, with Sobel Canny edge detection (SCED) algorithms to detect quickly and accurately water levels in real-time. After the water level is measured, a flood detection process is carried out based on the specified water level. According to the results of experiments in the laboratory, it has been shown that the proposed approach can detect objects accurately and fast in real-time. Besides, from the water level detection experiment, good results were obtained. Therefore, the object detection system and water level can be used as an efficient and accurate early detection system for flood disasters.
\end{abstract}

This is an open access article under the CC BY-SA license.

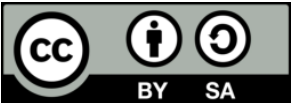

\section{Corresponding Author:}

Satriyo Budi Utomo

Department of Electrical and Computer Engineering

Universitas Jember

37 Kalimantan St., Kampus Tegal Boto, 68121, Indonesia

Email: satryo@unej.ac.id

\section{INTRODUCTION}

Flood disasters are natural disasters that often occur in areas with very high rainfall capacities. The rainfall is due to the many external and internal factors of nature itself, such as extreme weather, illegal logging, irrigation blockages, and others. Massive losses in the affected areas occur due to poor disaster management. According to [1] and [2], the use of an early warning system is significant in disaster management. Warnings carried out as an early warning system based on measurements of water level in rivers and dams.

Several kinds of Researches to measure water levels has accomplished in a variety of applications, methods, and locations. Several types of detection devices developed in research [3] to measure water level. Detection of water level measurements using a water gauge was done in mountainous areas as well [4], [5] and tidal [6]. The method of data acquisition has also been carried out both in real-time [7] and not in realtime [8]. Water level measurement techniques have performed using ultrasonic sensors [9], radar waves [10], and camera sensors [11]. The research revealed the computation system with the convolutional neural network (CNN) method [12] based on IoT [13], [14]. Preparation of the system for early warning of disasters using the system for smart cities in predicting the occurrence of floods [15] then the use of sensors in making 
intelligent river systems that can control the rate of water in monitoring when flooding occurs. The use of internet of things (IoT) in providing an early warning when a flood occurs [16] based on an artificial neural network, and there is a time limit to minimize the impact of mitigation when a flood occurs.

The integration of image processing and computer vision is a method that is currently have developed in various detection applications. Several studies have proven that image processing and edge detection methods applied for object detection [17]-[19] and recognition of particular objects or events [20]. The use of closed-circuit television (CCTV) camera analysis has been carried out [21]. The background subtraction algorithm used to detect the level of water experiencing movement to a high and unsafe position. The monitoring process at the water level using an IoT-based camera was also developed [22]. The flood detection process is sent via the internet and sent to each user in the flood detection process only explains the prototype in sending flood data using solar cells as a power supply from the camera. The process of automatically detecting floods with the segmentation method under flood conditions is discussed [23]. The automatic process carried out using IoT in the process of sending flood detection signals used sent to each user. When water conditions have a color that exceeds the reasonable amount, flooding detected through a camera. However, the water conditions were not explained in detail when floods occurred. The flood detection process using variations in the movement of a single camera has also been estimated by [3] with image processing and photogrammetry techniques. But there is no explanation yet about efforts to warn against floods. The use of tools in the form of water level detection with a water level mark on the canal is processed using region-of-interest (ROI), and color segmentation of the water level indicator at the dam has been carried out by [24]. This process requires clear color indicators in the method of detecting the water level.

The problem that occurs is that much of the above research about early detection of floods using a camera but has not discussed the speed and accuracy of water level measurement. The measurement causes an early warning to be less effective in disaster management because flood disaster management requires fast and accurate decision making. The use of edge detection methods on the ball with the Sobel Canny edge detection (SCED) algorithm method carried out because it allows the measurement of water level carried out quickly and accurately based on the rise of the ball floating on the surface of the water. Therefore, the use of height gauge indicators and camera sensors is needed.

\section{RESEARCH METHOD}

\subsection{Experimental setup}

In the research, two experiments were performed, namely, the first experiment to find out the effectiveness of the method in object recognition and the second experiment for measuring water level with a warning level. The object recognition experiment as a water level indicator carried out using an orange ball placed in a container filled with water next to the water level gauge, as shown in Figure 1. The second experiment has set by placing the orange ball indicator on a bounding box filled with water added to the container for 61 minutes. Water level detection experiment for early warning is done by determining the height of the water level to three levels, namely safe level at water level $1-10 \mathrm{~cm}$, the alert level at water level $10-23$, and hazard level at the height of $24-27 \mathrm{~cm}$. The measurement was made with a water level gauge that was placed parallel to the bounding box, as illustrated in Figure 2.

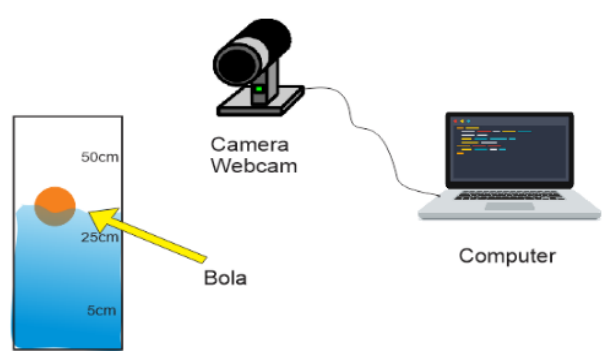

Figure 1. Experimental setup for object detection

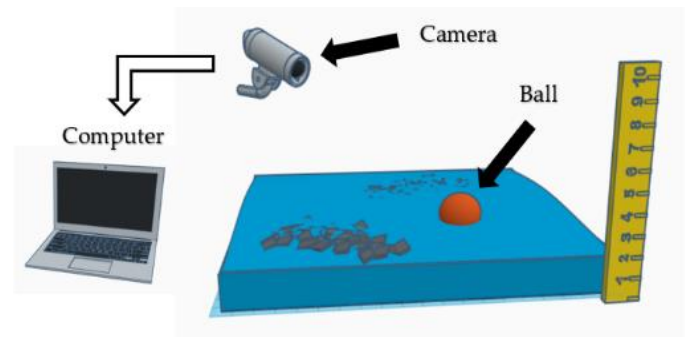

Figure 2. Experimental set-up for flood detection

\subsection{Water level recognition method}

A ping pong ball has been applied to monitor the water level and placed into the bounding box filled by the water. The ball was floating on the water surface and detected by the camera sensor, as described in Figure 1.The presence of the ball in the water used as an level indicator. Then the water level is classified into three signs for early warning of floods, namely safe, alert, and danger. The camera sensor used is LOGITECH webcam B525, with a resolution of $1280 \times 720$ pixels. After the camera detects the ball in the 
water, then image processing is computed. The software used is Python 3.1 with the OpenCV library as a database for the detection and segmentation and convolution process in the video camera.

In this study, image processing has employed in each frame for the detection process. In the initial stage, the color segmentation has done from red-green-blue (RGB) to hue-saturation-value (HSV) then the image is converted into grayscale. The next step is masking, where this process used to determine edge detection. Video input performed in the pre-processing stage in which the video transformed into a frame. The results of processing in each frame are processed by converting an RGB image to HSV and then turned to a grayscale image, as seen in the flow diagram of Figure 3, to be able to detect objects in each frame.

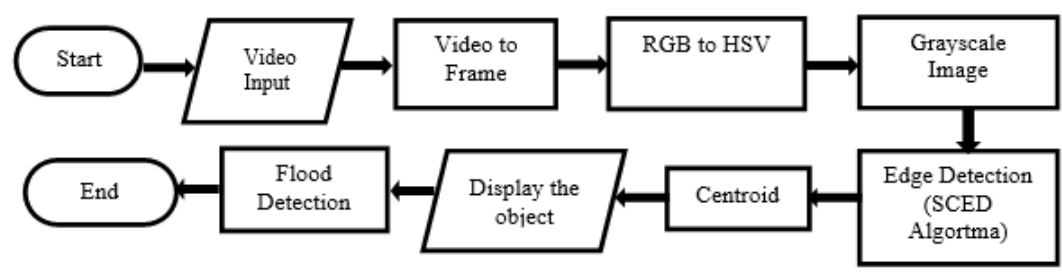

Figure 3. Flowchart flood detection process

\subsubsection{Convolution}

The purpose of convolution is to convert images from RGB to grayscale. Convolution is a process in which images manipulated using external masks/subwindows to produce new images. Red, green, blue (RGB) color space is a combination of primary colors, namely red, green, and blue, commonly used by computer monitors or televisions. The resulting color comes from a combination of three colors, and each has a value of eight red bits, eight green bits, and eight blue bits. Mixing the three primary colors with an equivalent fraction will produce shades of gray. If all three colors are fully saturated, it will produce white.

\subsubsection{Segmentation}

Image segmentation is a process that aims to separate the foreground region from the background region. This separation based on the striking differences in the characteristics of each area. As for the image segmentation process itself, there are several algorithms, consisting of point, line, and side detection algorithms (based on Robert operator and Sobel operator). In [19] states that segmentation is the process of dividing an image into several parts or objects. Segmentation has a very significant part of automatic image analysis because, in this procedure, the desired object will be tapped for the next process, for example: in pattern recognition. The segmentation algorithm based on two characteristics of the degree of image brightness, namely: disruption and conformity [25]. In the first item, the image is separated/divided based on a striking change from the degree of brightness. Typical applications are for the detection of points, lines, areas, and sides of an image. In the second category, it based on thresholding, region growing, and region splitting and merging. The principle of image segmentation applied to static and dynamic images. Image segmentation is dividing an image into homogeneous parts.

\subsubsection{Edge detection}

Edge detection has an important role to detect edges that limit two homogeneous image regions that have different brightness levels. The aim is to convert 2D images into curved shapes. The side is some part of the image where the brightness intensity changes drastically [25]. The critical factor in extracting features is the ability to detect the presence of edges of objects in the image. Edge detection becomes the first step to encompass information in the picture. Edges characterize the boundaries of objects and are therefore useful for segmentation and identification in the vision. Besides, the purpose of edge detection is to increase the appearance of the boundary lines of an area or object in the picture.

a. Canny operator

There are five steps taken to implement Canny edge detection, namely:

- Step1 is filtering of the image is done to eliminate noise using a Gaussian filter with simple levels with the provisions that the standards used are much smaller than the size of the picture.

- Step 2 is that after smoothing the image against noise is done, then the process of getting edge strength (edge strength) using the Gaussian operator. Image gradients calculated using formula 1.

$$
|G|=|G x|+|G y|
$$

- Step 3 is to calculate the edge direction. The formula used is as shown in (2). 


$$
\text { theta }=\tan -1(G x G y)
$$

- Step 4 is to connect the edge direction with an image traceable path.

- Step 5 is the Hysteresis process, i.e., eliminating broken lines.

Based on the explanation above steps, to detect edges with the Canny method, we will use a gradient $\mathrm{G}(\mathrm{x}, \mathrm{y})$, which is a vector consisting of two elements, namely Gx and Gy. Edge detection is done by rendering each pixel in the image by learning from the top leftmost pixel (northeast) and moving to the bottom rightmost pixel (southwest). Therefore, to assist with edge tracing, the gradients of Gx and Gy are calculated respectively with the 3x3 Canny mask operator matrix, as depicted in Figure 4 .

b. Sobel operator

Similar to Canny, the gradient G (x,y), which is a vector made of two elements, Gx and Gy, is used to detect edges by the Sobel method. Edge detection is done by reading each pixel on the image by learning from the top-left pixel (northeast) and moving to the bottom rightmost pixel (southwest). Therefore, to help trace the edges, the gradients of Gx and Gy are calculated using the 3x3 Sobel Mask matrix method. This method takes the principle of the laplacian and gaussian functions known as functions to generate HPF. The advantage of this Sobel method is the ability to reduce noise before performing edge detection calculations. The constant $\mathrm{C}$ value is two, so a Sobel operator matrix formed, as shown in Figure 5.

\begin{tabular}{|c|c|c|c|c|c|c|c|}
\hline \multirow{3}{*}{$C_{x}$} & 1 & 0 & -1 & \multirow{3}{*}{$C_{y}$} & -1 & -1 & -1 \\
\hline & 1 & 0 & -1 & & 0 & 0 & 0 \\
\hline & 1 & 0 & -1 & & 1 & 1 & 1 \\
\hline
\end{tabular}

Figure 4. Canny operator

$S x=$\begin{tabular}{|c|c|c|}
\hline-1 & 0 & 1 \\
\hline-2 & 0 & 2 \\
\hline-1 & 0 & 1 \\
\hline
\end{tabular}

$S y=$\begin{tabular}{|c|c|c|}
\hline 1 & 2 & 1 \\
\hline 0 & 0 & 0 \\
\hline-1 & -2 & -1 \\
\hline
\end{tabular}

The logic design applied the unified modeling language (UML). It consists of a use case design for image segmentation, Canny's operator, and Sobel's operator. The physical model describes the design of the main form, operator Canny and operator Sobel form.

c. SCED algorithm

Sobel Canny edge detection (SCED) algorithm is a new method in edge detection that combines the Canny and Sobel techniques. The result is an original method that provides excellent results and less noise. The concept of SCED matrix a logarithm is quite simple, namely, just take the $\mathrm{X}$ component of the Canny matrix and then combine it with the Y component of the Sobel method. The purpose of this method is to mix and complete the advantages and disadvantages of the two methods, the Canny and Sobel methods. An explanation of the SCED algorithm method has been shown in (3), (4) and (5).

- Formula 1. Horizontal Side in SCED

$$
E x=C x=\left[\begin{array}{lll}
1 & 0 & -1 \\
1 & 0 & -1 \\
1 & 0 & -1
\end{array}\right]
$$

- Formula 2. Vertical Side in SCED

$$
E y=S y=\left[\begin{array}{ccc}
-1 & -2 & -1 \\
0 & 0 & 0 \\
1 & 2 & 1
\end{array}\right]
$$

- Formula 3. SCED Matrix

$$
\left.\begin{array}{crc}
\text { Ex } & \multicolumn{3}{c}{\text { Ey }} \\
1 & 0 & -1 \\
1 & 0 & -1 \\
1 & 0 & -1
\end{array}\right]\left[\begin{array}{ccc}
-1 & -2 & -1 \\
0 & 0 & 0 \\
1 & 2 & 1
\end{array}\right]
$$

The results of combining the two Ex and Ey automatic will be combined using the gaussian operator used in step two of the Canny operator to get the edge value from each existing pixel distribution.

\subsection{Centroid}

The centroid is the arithmetic mean value of an object's shape from all points in the object. The centroid position of the object is treated as the basic measure value or the Eigen value. So, to determine the midpoint of an object used centroids in the detection process [7]. 


\subsection{OpenCV}

OpenCV stands for open computer vision, an open-source library that is devoted to image processing. OpenCV has the advantage of being a multi-platform framework, supports windows and linux, and, more recently, Mac OS X. There are many edges detection functions available in OpenCV, including cvCanny, cvLaplace, and cvSobel. [4].

\section{RESULTS AND DISCUSSION}

This algorithm has used to combine two operator functions where the Sobel operator and the Canny operator are used to detecting edges of the image after image segmentation performed. SCED Alogarithm itself is something that can raise the possibility of detecting an object with a high level of detection accuracy. By combining the horizontal matrix values of the Canny operator and the vertical matrix for the Sobel operator matrix.

\subsection{Ball detection}

Detection of the ball was carried out through the detection process using the algorithm SCED method. In digital image pre-processing [15], the image convolution process handled by changing the RGB image to grayscale. Next to the segmentation stage with masking techniques, edge detection is done using the SCED algorithm. The results of masking detection showed the ball could form a circle, even though the shape of a circle is not continuous. The existence of the water surface changes the form of the floating ball. The change is because the edge of the ball detected is the result of image segmentation when going through the process of changing the grayscale image, as seen in Figure 6. Then the results of the detection are used to determine the mean value of the circle object created so that the object can be displayed.

The detection process uses the color of the ball with the orange line background by adjusting the detection. The results of the application of detection only detect colors on the ball because the number of orange pixels on the ball and back region have different resolutions. This part caught in the image is just the color of the ball by forming the edge of a circle. Meanwhile, the area was not detected due to not meeting the pixel resolution threshold value. The background is not recognized because it does not reach the pixel resolution threshold. The experiment was done as well by bringing the ball closer to the background color, and the results of the test shape show the ball resolution increases. Adjustment of the segmentation color in the background causes the detection to adjust to the value of the background edge and is considered to be integrated with the ball so that this detection is not well done.

A further experiment was done by differentiating the results from the pixel distribution graph when the ball is detected using the SCED algorithm method. The vulnerable 150-170 colors have a very high level of pixel distribution, as shown in Figure 7, where the x-axis (horizontal) is the number of pixel distributions and the graph y (vertical) is the number of color variants found in the RGB pixel. The pixel distribution is because the orange color variations on the ball are very bright. The gap in the graph of the histogram value is an area that does not have pixel probability because the type and size of the pixel can affect the color of the pixel. The higher the pixel resolution used, the fewer pixel distribution gaps.

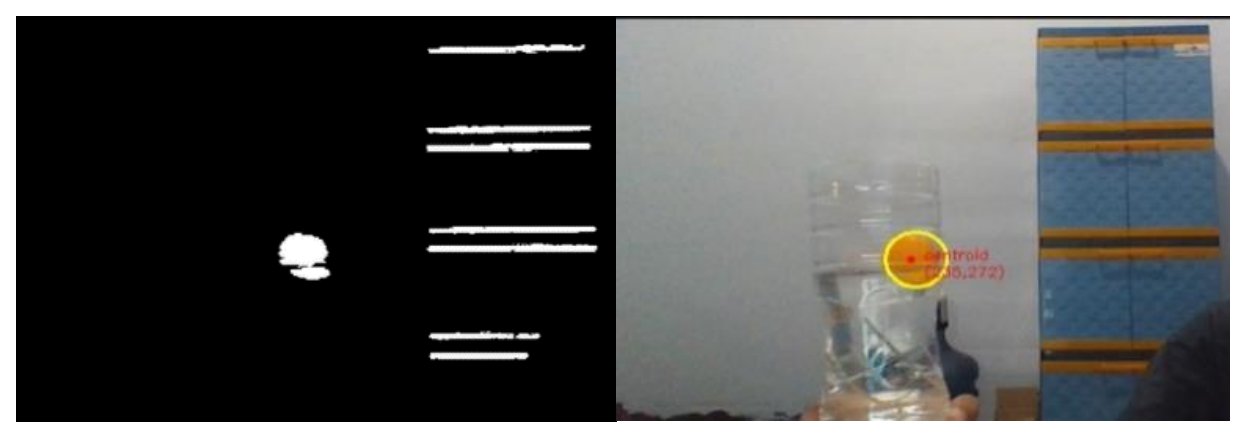

Figure 6. Mask detection results

Similarly, binary images that have values 1 and 0 , very little pixel distribution after the segmentation process to grayscale color. The use of segmentation can reduce the level of pixel distribution that exists in the image, as seen in the histogram value in Figure 8. This is because only white and black are scattered. With a very minimal amount of color probabilities, the detection of objects from an image can be done rapidly. 


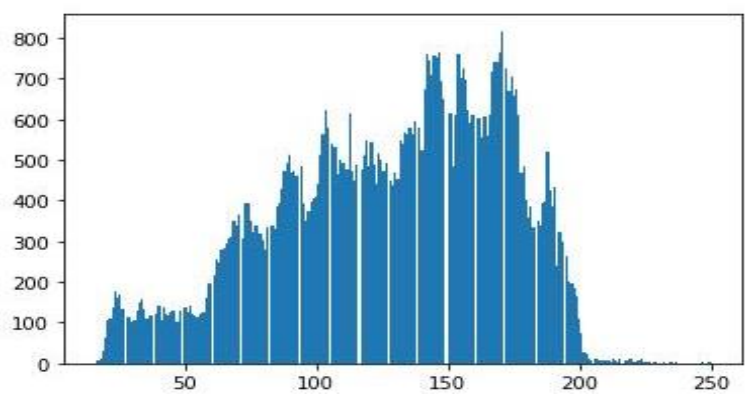

Figure 7. Histogram values for detecting pixel spheres

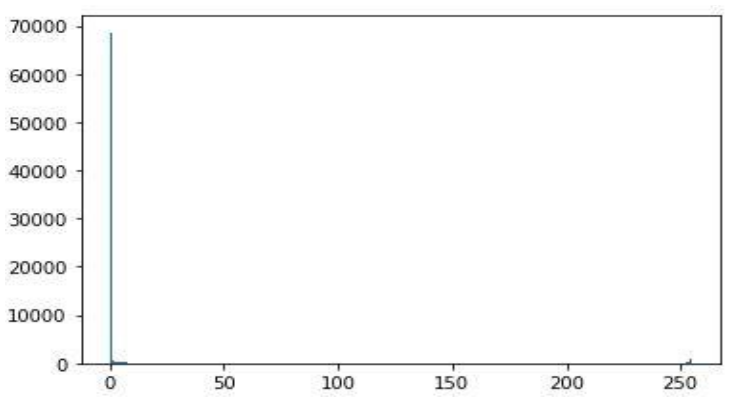

Figure 8. Pixel histogram detection value in the masking process

\subsection{Water level and flood warning recognition}

In this experiment, a water level gauge is used to indicate the water level as a flood occurs. The test is carried out by determining the level of the water level at the height of 1-10 cm, 11-23 cm, 24-27 as secure, an alert, the danger, respectively. By determining the average value of the object centroid, pixel resolution measurements were used to adjust the shape of the container used. Each pixel bounding box and the line in the height gauge was conformed to the pixel position of the container shape $\mathrm{F}(\mathrm{x})$ and $\mathrm{F}(\mathrm{y})$ to the image digital. Based on observation Table 1, it was found that the detection of the sphere is by the condition of the water level, which is safe at $1-10 \mathrm{~cm}$ and alert conditions $11-23 \mathrm{~cm}$ and hazard conditions at $24-27 \mathrm{~cm}$. In this experiment, the time elapsed is less than 1 minute, and the ball indication can detect the water level accurately. This can be indicated by the movement of water that rises in the container when the centroid value of the ball reaches the pixel line on the water level gauge. Indications that occur at the time of detection are following specified values. When the centroid reaches the line, it will be true, and when it passes through a different route than before, it will be False. And when the conditions in the next bounding box line are correct.

Table 1. Water level and flood warning experimental results

\begin{tabular}{cccc}
\hline No. & Time Elapsed & Water Level Observed at Water Level Gauge $[\mathrm{cm}]$ & Measurement Result of Warning on Computer \\
\hline 1 & $13: 00$ & 1 & Secure \\
2 & $13: 01$ & 2 & Secure \\
3 & $13: 02$ & 3 & Secure \\
4 & $13: 04$ & 4 & Secure \\
5 & $13: 07$ & 5 & Secure \\
6 & $13: 12$ & 6 & Secure \\
7 & $13: 15$ & 7 & Secure \\
8 & $13: 19$ & 8 & Secure \\
9 & $13: 25$ & 9 & Secure \\
10 & $13: 27$ & 10 & Secure \\
11 & $13: 30$ & 11 & Alert \\
12 & $13: 32$ & 12 & Alert \\
13 & $13: 33$ & 13 & Alert \\
14 & $13: 35$ & 14 & Alert \\
15 & $13: 37$ & 15 & Alert \\
16 & $13: 38$ & 16 & Alert \\
17 & $13: 40$ & 17 & Alert \\
18 & $13: 44$ & 18 & Alert \\
19 & $13: 45$ & 19 & Alert \\
20 & $13: 48$ & 20 & Alert \\
21 & $13: 49$ & 21 & Alert \\
22 & $13: 50$ & 22 & Alert \\
23 & $13: 51$ & 23 & Alert \\
24 & $13: 52$ & 24 & Danger \\
25 & $13: 55$ & 25 & Danger \\
26 & $13: 59$ & 26 & Danger \\
27 & $14: 01$ & 27 & Danger \\
\hline & & &
\end{tabular}

\section{CONCLUSION}

The use of the SCED method showed that the ball of the same color as that of the ball could affect noise in detection. However, by limiting the number of pixel distributions, the ball object detection process can be optimized. Therefore, the proposed approach can detect water level and differentiate alert indicators for flood early warning. Based on the Flood detection experiment, it showed that the SCED algorithm method could 
provide accurate and fast measurements to determine conditions when water level changing occurs. Besides, the flood detection through water level can be done in less than 1 minute with the same results as specified.

\section{REFERENCES}

[1] J. Mulyono, "The three pillars: deliberative coordination management model (in mitigating flash flood and landslide disasters in Jember Regency)," Proceeding Book Vol. 2, 2019.

[2] A. Wardhono, M. Rondhi, J. F. Irawan, and B. Prakoso, "Identification and mapping of disaster risk of flash floods in Jember Regency-East Java, Indonesia," International Symposium, Microdis and Hue University, Vietnam, 2010.

[3] Y.-T. Lin and Y.-C. LinAsada, "Automatic water-level detection using single-camera images with varied poses," Measurement, vol. 127, pp. 167-174, 2018, doi: 10.1016/j.measurement.2018.05.100.

[4] E. Ridolfi and P. Manciola., "Water level measurements from drones: a pilot case study at a dam site," Water, vol. 10, no. 3, pp. 297, 2018, doi: 10.3390/w10030297.

[5] Y. H. Zhang., "A brief discussion on model selection of water level gauge for mountain river," Autom.Water Resources Hydrology, pp. 45-46, 2008.

[6] Y. Zhang and Q. Shen, "Application and discussion of ultrasonic and float type water level gauge in sluice pump station of tidal river," Jiangsu Water Resources, vol. 6, pp. 6-9, 2017.

[7] T. M. Thekkil, et al., "Real-time WSN based early flood detection and control monitoring system," 2017 International Conference on Intelligent Computing, Instrumentation and Control Technologies (ICICICT), 2017, doi: 10.1109/ICICICT1.2017.8342828.

[8] B. B. Nair and S. Rao., "Flood Water Depth Estimation-A Survey," IEEE International Conference on Computational Intelligence and Computing Research, pp. 1-4, 2017, doi: 10.1109/ICCIC.2016.7919573.

[9] T. M. Tsai and P. H. Yen., "Improvement in stage measuring technique of the ultrasonic sensor gauge," Measurement, vol. 45, no. 7, pp. 1735-1741, 2012, doi: 10.1016/j.measurement.2012.04.012.

[10] G. B. Li, et al., "Application of guided-wave radar water level meter in tidal level observation," Journal Ocean Technol, vol. 37, pp. 19-23, 2018.

[11] J. Yu and H. Hahn., "Remote detection and monitoring of a water level using narrow-band channel," Journal of Information Science and Engineering, vol. 26, pp. 71-82, 2010.

[12] M. Anbarasan, B. A. Muthu, and Sivaparthipan, "Detection of Flood Disaster System Based on IoT, Big Data and Convolutional Deep Neural Network," Computer Communications, vol. 150, pp. 150-157, Aug 2019, doi: 10.1016/j.comcom.2019.11.022.

[13] S. Sood, K. R. Sandhu, K. Singla, and V. Chang., "IoT, big data and HPC based smart flood management framework," Sustainable Computing: Informatics and Systems, vol. 20, pp. 102-117, 2018, doi: 10.1016/j.suscom.2017.12.001.

[14] A. Sinha, P. Kumar, N. P. Rana, R. Islam, and Y. K. Dwivedi, "Impact of the internet of things (IoT) in Disaster Management: A task-technology fit perspective," Ann. Oper. Res, pp. 1-36. 2017, doi: 10.1007/s10479-017-2658-1.

[15] G. Furquim, R. Jalali, G. Pessin, R. Pazzi, and J. Ueyama, "How to improve fault tolerance in disaster predictions: A case study about flash floods using IoT, ML, and real data," Sensors, vol. 18, no. 3, pp. 907, 2018, doi: $10.3390 / \mathrm{s} 18030907$.

[16] N. Maspo, et al., "Development of Internet of Thing (IoT) technology for flood prediction and early warning system (EWS)," Int. J. Innov. Technol. Explore. Eng, vol. 8, pp. 219-228, 2018.

[17] T. De Marco, and D. Cazzato, "Randomized circle detection with isophotes curvature analysis," Pattern Recognition, vol. 48, no. 2, pp. 411-421, 2015, doi: 10.1016/j.patcog.2014.08.007.

[18] M. Aqil, M. Fuad, M. Ruddin, A. Ghani, and Tole Sutikno., "A Review on Methods of Identifying and Counting Aedes Aegypti Larvae using Image Segmentation Technique," TELKOMNIKA Telecommunication, Computing, Electronics and Control, vol. 15, no.3, pp. 1199-1206, 2017, doi: 10.12928/telkomnika.v15i3.6422 .

[19] R. Muthukrishnan and M. Radha., "Edge detection techniques for image segmentation", International Journal of Computer Science \& Information Technology, vol. 3, no. 6, pp. 259-267, 2011, doi: 10.5121/ijcsit.2011.3620.

[20] V. Wiley and T. Lucas., "Computer vision and image processing: A paper review," International Journal of Artificial Intelligence Research, vol. 2, no. 1, 2018, doi: 10.29099/ijair.v2i1.42.

[21] M. J. P. S. Miguel, and C. R. Ruiz., "A flood detection and warning system based on video content analysis," Springer International Publishing AG, 2016, doi: 10.1007/978-3-319-50832-0_7.

[22] C.-H. Tseng, and L.-C. Chen., "An automated image dehazing method for flood detection to improve flood alert monitoring system," Journal of the National Science Foundation of Sri Lanka, vol. 46, no. 3, pp.329-339, 2019, doi: 10.4038 /jnsfsr.v46i3.8485.

[23] R. H. Randhawa, and R. Mahmood, "AquaEye: A low-cost flood early warning system for developing countries," Int. Conference on Frontiers of Information Technology, pp. 345-349, 2018, doi: 10.1109/FIT.2018.00067.

[24] Y. M. Akbar, A. Musafa, and I. Riyanto., "Image Processing-based Flood Detection for Online Flood Early Warning System," Indonesia Japan Joint Scientific Symposium, 2014.

[25] J. Cai, H. Panfeng, L. Chen, B Zhang., "An efficient circle detector not relying on edge detection," Advances in Space Research, vol. 57, no. 11, pp. 2359-2375, 2016, doi: 10.1016/j.asr.2016.03.026. 